\title{
High Energy Efficient Heterogeneous Networks: Cooperative and Cognitive Techniques
}

\author{
Heng Wang, ${ }^{1,2}$ Jiamo Jiang, ${ }^{2}$ Jian $\mathrm{Li}^{2}{ }^{2}$ Manzoor Ahmed, ${ }^{2}$ and Mugen Peng ${ }^{2}$ \\ ${ }^{1}$ Department of Management Science and Engineering, School of Economics and Management, Tsinghua University, \\ ShuangQing Road 30, Beijing 100084, China \\ ${ }^{2}$ Key Laboratory of Universal Wireless Communications of Ministry of Education, Wireless Signal Processing \& Network Lab, \\ Beijing University of Posts and Telecommunications, XiTuCheng Road 10, Beijing 100876, China
}

Correspondence should be addressed to Heng Wang; wangheng_2003@tsinghua.org.cn

Received 7 November 2013; Revised 23 November 2013; Accepted 29 November 2013

Academic Editor: Xiang Zhang

Copyright (C) 2013 Heng Wang et al. This is an open access article distributed under the Creative Commons Attribution License, which permits unrestricted use, distribution, and reproduction in any medium, provided the original work is properly cited.

Heterogeneous network (HetNet) is considered as the main and eminent future communication technology, since it achieves high spectral efficiency per unit area and saves energy due to low transmission power. Mass deployment of small cells in cochannel mode increases overall system capacity, but it is also coupled with greater risk of cochannel interference. This paper overviews the interference model based on the Poisson point process (PPP) and analyzes the performance in terms of energy efficiency in multitier HetNet. As the promising solution for improving the performance of HetNet, both the cooperative communication and cognitive radio techniques to mitigate the interference in HetNet are surveyed. As one example of cooperative communication techniques, a hierarchical cooperation scheme on the spectrum allocation is presented and its energy efficiency performance is analyzed and evaluated. Meanwhile, the energy efficiency increases from the cognitive radio technique are demonstrated as well. The energy efficiency performance comparison between the presented cooperative communication and cognitive radio techniques is emphasized, which suggests that the cooperation communication technique is preferred to suppress the interference and increase the energy efficiency in HetNets.

\section{Introduction}

Heterogeneous network (HetNet), which consists of a mix of generous macrocells and low-power nodes, for example, micro, pico, femto, relay node (RN), and remote radio head $(\mathrm{RRH})$, has been seen as a promising approach for improving the spectrum and energy efficiency. The large macrocells provide mobility and basic coverage, while small cells and low-power nodes boost capacity and extend the range of cellular network [1]. Meanwhile, the environmental effect of the information and communication technologies (ICTs) has become an increasingly hot topic, which is known that the ICTs have been a major contributor to total greenhouse gas emissions. Not only the government, but also the operators pay a great attention to reduce the capital expenditure (CAPEX) and operational expenditure (OPEX) by saving the energy consumption with advanced wireless communication techniques. In HetNet, the coexistence of macro- and small cells has the potential to reduce the energy consumption by shortening the distance between the user equipments (UEs) and eNodeBs (eNBs) [2].

However, the HetNet architecture also brings new issues and challenges compared with traditional homogeneous network, such as the impact of resource allocation and interference mitigation schemes on the performance [3]. For orthogonal resource allocation scheme, the interference is avoided by sacrificing the available resources, while for the cochannel resource scheme, the spectrum efficiency improves under the expense of increasing the interference. Meanwhile, there is serious impact of interference on the energy efficiency. Therefore, advanced transmission techniques and interference managements need to be proposed and designed carefully. Cooperative and cognitive schemes have attracted much attention in the design of interference management for homogeneous network to enhance the performance in terms of spectrum and energy efficiency [4]. For the cooperative 
schemes in homogenous and heterogeneous networks, different UEs or eNBs are allowed to share resources and channel information to implement collaboration. For the cognitive schemes in homogenous and heterogeneous networks, cognitive radio technique enables the utilization of adaptive and intelligent transmission strategies with a distributed transmission manner.

In order to increase the resource utilization and overall system capacity, macro- and small cells are deployed in cochannel mode in HetNet usually, while it has to couple with greater risk of intertier and intratier interferences. A significant amount of research has been done to manage the cochannel interference (CCI) in a two-tier network, comprising a macrocell network overlaid with small cells [5]. In this regard, much related research has focused on ways to investigate the interference, manage the resource, and improve the spectrum efficiency with the consideration for cooperative or cognitive schemes in HetNet. On one hand, in contrast to traditional noncooperative communications, different users and base stations are allowed to share the resources and to implement collaboration by information exchange in cooperative networks. A hierarchical spectrum management concept which can coordinate resource allocation for both licensed and unlicensed bands in HetNet is proposed in [6]. A hierarchical cooperative relay-based HetNet to support both unicast and multicast services is proposed to provide a cost-effective coverage extension with the development of heterogeneous relay nodes [7]. On the other hand, cognitive radio can play its role as defined by Mitola and Maguire [8], where wireless node or network takes into account every possible measurable parameter to intelligently alter its functionality to meet a certain goal and one of these goals can be power saving. Interference can be managed by applying cognitive resource allocation schemes and several literatures focus on such resource allocation especially in the cellular cognitive radio. Furthermore, there is a firm motivation to introduce cognitive abilities to small cell eNBs (SeNBs) and adapt to their behaviors according to the dynamic changes in the environment, such as number of UEs and number of eNBs. However, majority of the current interest is focused on the problem of two-tier HetNet interference management and somehow neglected the dimension of energy efficient issue in the HetNet.

However, to the best of our knowledge, the energy efficiency comparison between the cooperative and cognitive schemes in HetNet has not been studied much in related works. This motivates us to write this paper to investigate the energy efficiency comparison of cooperation and cognition in HetNet. In addition, the energy efficient transmission schemes are proposed with cooperative and cognitive techniques. Similarly, interference model in HetNet is presented to investigate the impact of interference on the performance of HetNet, and the energy efficiency improvements in HetNet achieved by interference cancelation and resource allocation schemes are analyzed. Lastly, the energy efficiency comparison of cooperation and cognition in HetNet is presented and some energy efficient transmission schemes are proposed, with the simulation results to evaluate the performance.
The remainder of this paper is organized as follows. The interference model of HetNet is investigated in Section 2, and a comprehensive comparison of the performance in HetNet is introduced in Section 3. In Section 4, the cooperative and cognitive schemes are proposed and the energy efficiency comparison between cooperative and cognitive schemes in HetNet is evaluated by simulation, followed by the concluding remarks regarding this paper in Section 5.

\section{Interference Model in HetNet}

The performance of users is degraded due to the cochannel interference in the orthogonal frequency division multiplexing (OFDM) cellular network where the frequency resources are shared between neighboring eNBs. The interference problem is essentially challenging in HetNets with respect to the limited backhaul capability supporting interference coordination between macro-eMBs (MeNBs) and SeNBs, the restricted access control (e.g., closed access femtocells), and the demand of monitoring cochannel interference from different types of nodes [9]. As shown in Figure 1, there are difference kinds of heterogeneous nodes located in an overlapping area. In order to investigate the interference management techniques in HetNet to provide energy efficient utilization of the bandwidth and enhance the cell edge user experience, the interference characteristics have to be studied in advance.

The major factors affecting the cochannel interference include the spatial distribution of interfering nodes and the propagation parameters. The spatial locations of homogeneous cellular nodes have been investigated in linear array models, square networks, triangle networks, and hexagon networks, where the topology is regular and the node locations are deterministic. In HetNet, however, the deployment of SeNBs is generally performed without careful network planning. Consequently, it is difficult to evaluate a tractable model for these randomly distributed nodes based on the aforementioned deterministic structures. To capture the random characteristic of spatial locations, a concise and tractable HetNet model has been recently discussed based on a point process, where the positions of eNBs are stochastically modeled and only the statistical description is available. The most widely used model is the Poisson point process (PPP), where the eNBs with intensity are uniformly distributed over the area. For the infinite planar network, the aggregate interference can be characterized with a shot-noise process, and the probability density function (PDF) is characterized by the intensity of eNBs instead of their specific locations. Extending this model to the HetNet, the eNBs in each tier can be modeled as independent PPP with different pathloss exponents and transmission power.

Although the interference is mainly determined by the positions of eNBs, the impact of propagation environment is nonnegligible. Rayleigh channel is the simplest fading model which results in an exponentially distributed receive power of a single link [10]. For the cellular network, since the aggregate interference is the sum of multiple links, the distribution characteristic is more complicated. For the model 


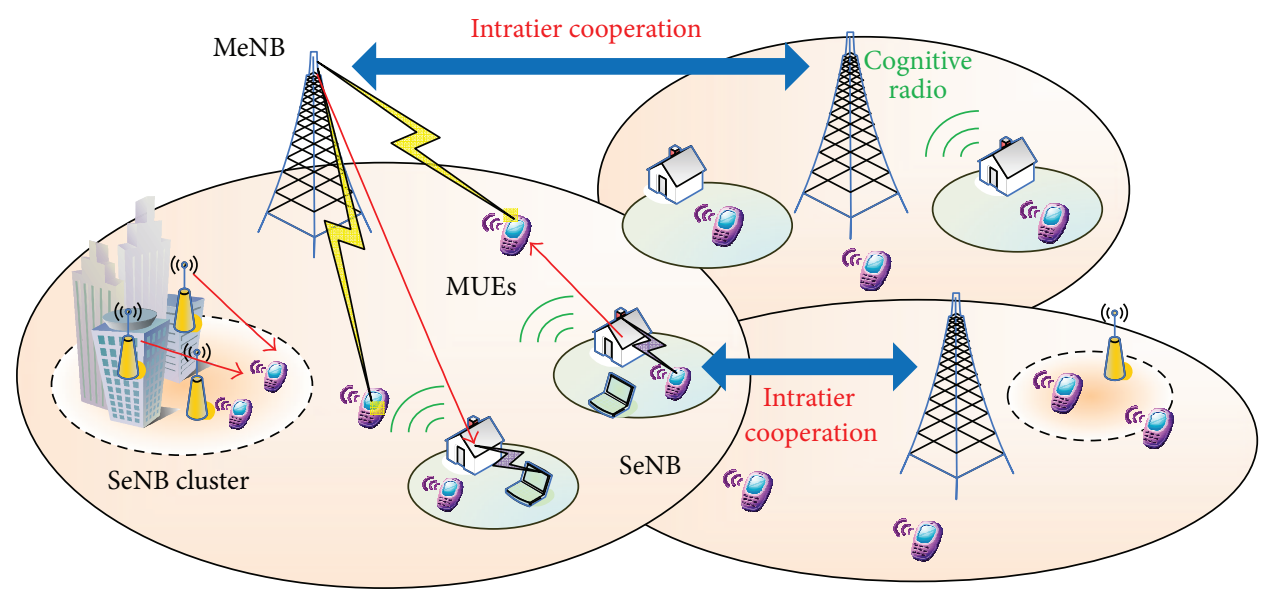

Figure 1: Transmission in multiple access relay channels.

of deterministic BS locations, the sum of interference can be expressed as a generalized chi-squared distribution, while a moment matching method is applied to approximate this sum of exponential distribution variables with a more concise gamma distribution [11]. In the stochastic model, it is feasible to incorporate various propagation effects such as shadowing and Nakagami-m fading, and the interference in the infinite plane can be captured as a skewed stable distribution when the pathloss exponent is larger than 2 [12]. A heavy tail property holds for the PDF of the stable distribution, which represents that the probability of closely located interfering nodes is not negligible.

\section{HetNet Performance Analysis}

Using the stochastic interference model, it is feasible to evaluate the performances of transmission reliability and capacity in large cellular networks. The success probability, defined as the probability that the signal to interference plus noise ratio (SINR) exceeds the predefined QoS threshold, is employed to evaluate the transmission robustness under the specific modulation and coding scheme (MCS). The capacity can be evaluated in terms of spatial average rate which denotes the mean rate of the instantaneous Shannon bound achieved by applying the adaptive MCS and area spectral efficiency which represents the network throughput achieved by using the specific MCS. The SINR for a two-tier HetNet with open access mode is depicted in Figure 2.

Andrews proposed a tractable framework for cellular networks, where the UE is associated with the nearest MeNBs. The distance of a UE to its serving MeNB can be obtained as a random variable according to the null probability of the Poisson distribution. Under this framework, the success probability and spatial average rate are derived for the homogeneous cellular network, which are observed to be independent with the density of MeNBs in the interferencelimited scenario. This framework can be easily extended to the HetNets with differing transmit powers, coverage areas, and access policies.

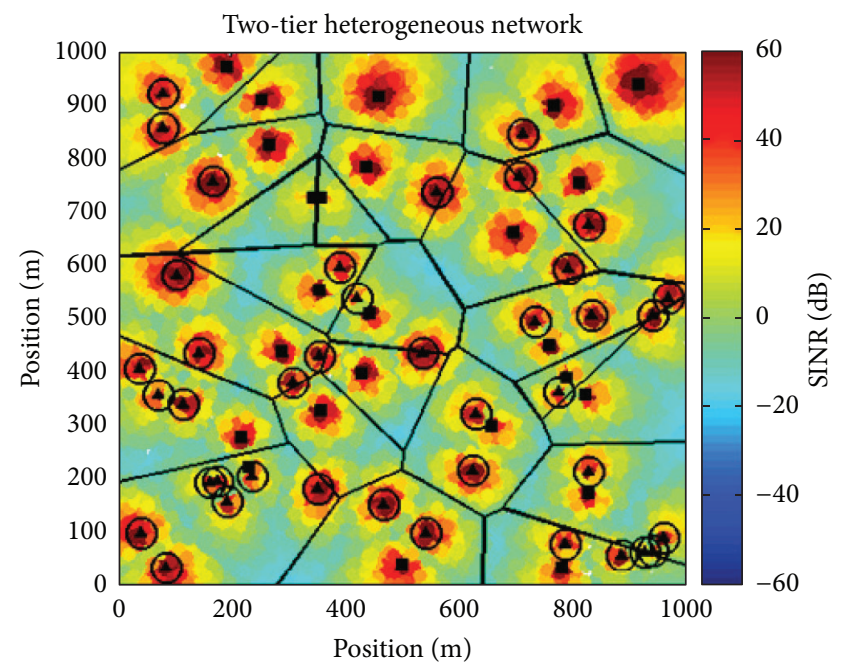

FIgURE 2: SINR for HetNets with open access mode.

In [13], the authors developed a flexible model for a Ktier HetNet, where eNBs in each tier are independently distributed differing in terms of transmit power, QoS threshold, and eNB density. The success probability and average load per tier were derived for the open access and closed access policies. The performance of the open access multitier HetNet was investigated in [14], where eNBs of each tier have a particular transmit power, pathloss exponent, density, and access bias. The performance was derived for a typical user in the whole network and associated with each tier, and the impact of the association bias was analyzed. According to the aforementioned literatures, it can be observed that the success probability of the unbiased multitier HetNet is not affected by the number of tiers or their relative densities in the interference-limited scenario when each tier has identical QoS threshold. On the other hand, adding more infrastructures of any type has a negligible impact on the coverage or throughput, so it is encouraging to provide throughput enhancement by increasing the number of eNBs, as 
more users could be served simultaneously by more eNBs. However, the resulting energy consumption is increased by deploying large amount of eNBs, which may lead to the degradation of energy efficiency.

The two-tier femtocell model was investigated in [15], where the FUEs are uniformly distributed in a thin ring centered around its serving femto access point (FAP). The success probability was evaluated for the macrocell and the femtocell in the shared subchannel and the unshared subchannel, and the optimal subchannel allocation was studied for the closed access policy and open access policy, respectively, to achieve the highest overall spectrum efficiency under the constraint of success probability and the spectrum efficiency of each tier. An opportunistic interference alignment scheme was proposed in [16], where a single MeNB configured with multiple transmit antennas is deployed underlaid with PPP distributed closed access MIMO FAPs. The performance was evaluated in terms of success probability, spatial average rate, spectrum efficiency, and energy efficiency, and the numerical results were shown to be superior over the scenario without interference alignment.

On the other hand, based on the definition of energy efficiency, we analyze the impacts of the average data rate, eNBs transmit power, and the circuit power on the energy efficiency of HetNet. The average rate increases with the eNBs transmit power and density. However, the increasing eNBs transmit power and density will also result in the increasing interference which will deteriorate the received signal SINR and the UE QoS requirement. The eNBs circuit power is the monotonically increasing function of the eNBs density which is the major part of the network power consumption. On the other hand, the circuit power largely depends on the bandwidth of eNBs transmit spectrum which will increase the operation power consumption of building baseband unit and remote radio unit. In wireless networks, the traffic volume from mobile users fluctuates in both temporal and spatial domains, which brings enormous challenges to energy efficient design of HetNet. Hence, the SeNBs sleep and zooming strategies are considered as the most promising method for reducing energy consumption while meeting UEs' increasing traffic demands.

\section{Energy Efficiency of Cooperative and Cognitive HetNet}

In HetNet, the conflict between the scarcity of spectrum resource and low resources utilization increases the energy consumption significantly and impairs the energy efficiency seriously. Because of the intertier and intratier interferences, the independent performance optimization in the homogeneous and isolated cell cannot converge to the global optimal solution of HetNet. Furthermore, the coordination schemes among the heterogeneous nodes have a strong impact on the complexity and performance of the network, which must be designed and carried out carefully.

In order to solve these challenges efficiently, the cooperation of the multidimensional radio resource and the cognition of the complicated scenario in HetNet should be investigated comprehensively. Cooperative and cognitive network are new paradigms in wireless communications to enhance the utilization of limited spectrum resources and improve the performance. Although the cooperative and cognitive radio networks can be integrated together and implemented jointly between different nodes in HetNet, a higher degree of cooperation and cognition brings a higher degree of complexity and cost in terms of network operation and energy consumption. In this section, the energy efficiency improvements of the proposed cooperative and cognitive HetNet are investigated and evaluated separately for low complexity. Then, the energy efficiency comparison of these two techniques is presented under different scenario and deployment of HetNet. Furthermore, the energy efficient networking schemes with different network parameters are proposed in HetNet.

\subsection{Energy Efficiency Improvements with Cooperative Het-} Net. In HetNet, there is two-tier cooperation employed to optimize the performance: intratier and intertier cooperation. These two cooperative structures can be deployed separately or jointly with the constraints on backhaul link and complexity. It is a highly desirable characteristic for interference-limited networks that full spectrum reuse is exploited to improve the data rate and increase the utilization of resource, especially in heterogeneous network where amounts of different nodes are deployed in an overlapping area. The energy efficiency versus the density of low-power nodes in HetNet is shown in Figure 3, when the spectrum resource is reused among MeNBs and SeNBs. In order to reduce the interference and improve the energy efficiency in HetNet, while increasing the resource utilization according to the load condition and small cells density, a hierarchical cooperation on the spectrum allocation is presented in this paper. Based on the load condition, received interference, and the number of small cells deployed in the network, the factor of spectrum reuse and the frequency allocation can be optimized among the heterogeneous nodes in a cooperative manner. By the framework of intratier and intertier cooperation, the proposed scheme is implemented in the following two steps.

In the first step, the intratier cooperation scheme is implemented among the MeNBs to reduce the intratier interference according to the fluctuation of MeNBs load. When there is some idle resource, the spectrum is allocated orthogonally among the MeNBs, which cause the most serious interference to others, by the cooperation schemes. In the second step, the intertier cooperation is explored between the MeNB and SeNB in the same macrocell to increase the utilization of spectrum while maintaining the QoS of macrocell UEs (MUEs). When the density of SeNBs is large, the reused frequency resource should be decreased for reducing the interference to MUEs. When there is small number of SeNBs located in the macrocell, more frequency resources can be reused for increasing the resource utilization efficiency. The reused spectrum resource should be optimized in a cooperated manner to decide the tradeoff between the impact of resource and interference on the energy efficiency. 


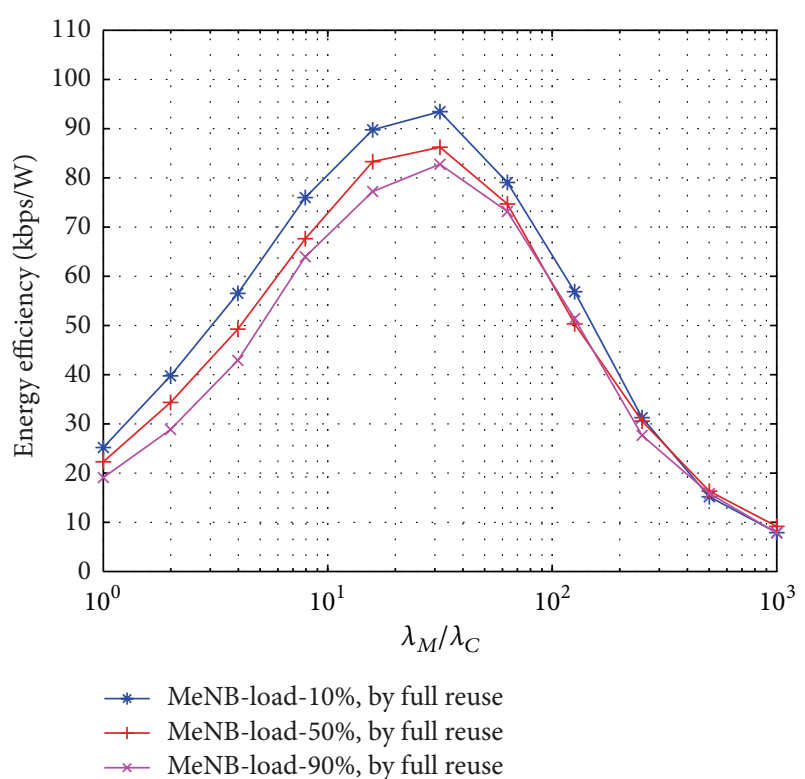

FIGURE 3: Energy efficiency in full reuse HetNet with different MBS load.

Figure 4 shows that the energy efficiency is improved by the cooperative scheme in HetNet. Compared to the energy efficiency in full reuse scheme depicted in Figure 3, higher energy efficiency can be obtained by the cooperation, especially when higher data rate requirement is supported in macrocells. It is claimed that the energy efficiency increases firstly and then decreases sharply with the increasing number of SeNBs. In a cooperative manner, the spectrum resource can be allocated adaptively among the heterogeneous nodes, by which higher utilization efficiency of resource is explored. When fewer SeNBs are deployed in the network, more frequency can be reused between the MeNB and SeNB. On the other hand, the energy efficiency can be improved significantly with a reasonable density of SeNBs when more resources are idle in MeNBs, because SeNB is more suitable in the energy efficient networking schemes.

4.2. Energy Efficiency Improvements with Cognitive HetNet. Cognitive radio $(\mathrm{CR})$ networks are designed to utilize the licensed spectrum when it is not used by the primary (licensed) users. The key enabling technologies of CR networks are the cognitive radio techniques that provide the capability to share the spectrum in an opportunistic manner. In traditional homogeneous network, the cognitive abilities have a wide range of potentials for improving the tradeoff among resources utilization, limited bandwidth, spectrum efficiency, and energy efficiency in future wireless communication network. The hierarchy in energy games for cognitive radio networks is investigated in [17], in which the energy efficiency for selfish secondary users is maximized. In [18], the sensing strategy (the switching point to stop sensing and begin secondary transmission) and power allocation scheme are designed jointly to optimize the energy efficiency.

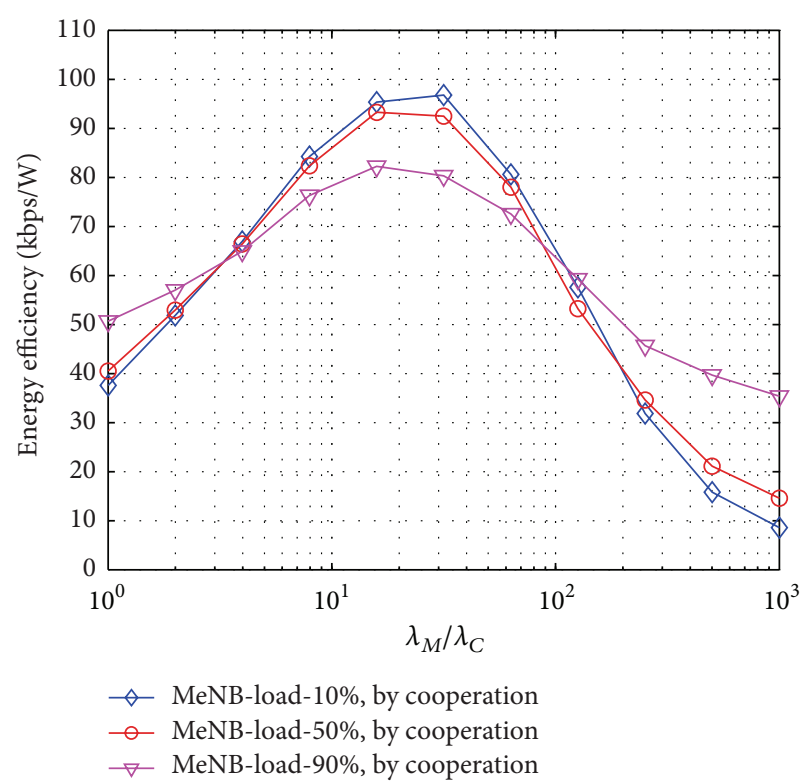

FIGURE 4: Energy efficiency in cooperative HetNet with different MBS load.

With the development of heterogeneous nodes, MeNBs which are located for basic coverage have priority in utilizing the spectrum to satisfy the requirement of user's QoS (as the primary users in common CR networks). If the SeNB can automatically sense the surrounding, the received signaling from the MeNB and other SeNBs (in the close vicinity), and intelligently allocate resources without any excessive intertier and intratier interferences, the network performance in terms of spectrum and energy efficiency will be enhanced significantly.

In cognitive HetNet, MeNBs are the licensed owner of the channel and SeNBs are allowed to seek transmission opportunities on the licensed spectrum of MeNB only when the macro-UEs are absent on these spectrum resources. It is assumed that these spectrum resources can be reused among the SeNBs. With this constraint, the intertier interference between MeNBs and SeNBs does not exist but the intratier interference among MeNBs or SeNBs cannot be neglected. Although more shared spectrum resources can be utilized with more cognitive SeNBs, the increasing intratier interference will impair the network performance seriously. The density of cognitive nodes is believed to be a crucial factor that affects the spectrum and energy efficiency of the HetNet, which cannot be neglected in the design of practical network with the consideration for the circuit power consumption and deployment cost.

As the simulation results illustrated in Figure 5, it is indicted that the energy efficiency is improved by the cognitive scheme in HetNet, especially when the traffic load of MeNBs is high. It is claimed that the energy efficiency increases firstly and then decreases sharply with the increasing number of SeNBs. Cognitive scheme is preferred with low traffic load of MeNB when there is small number of SeNBs in the network, because there are limited improvements of 


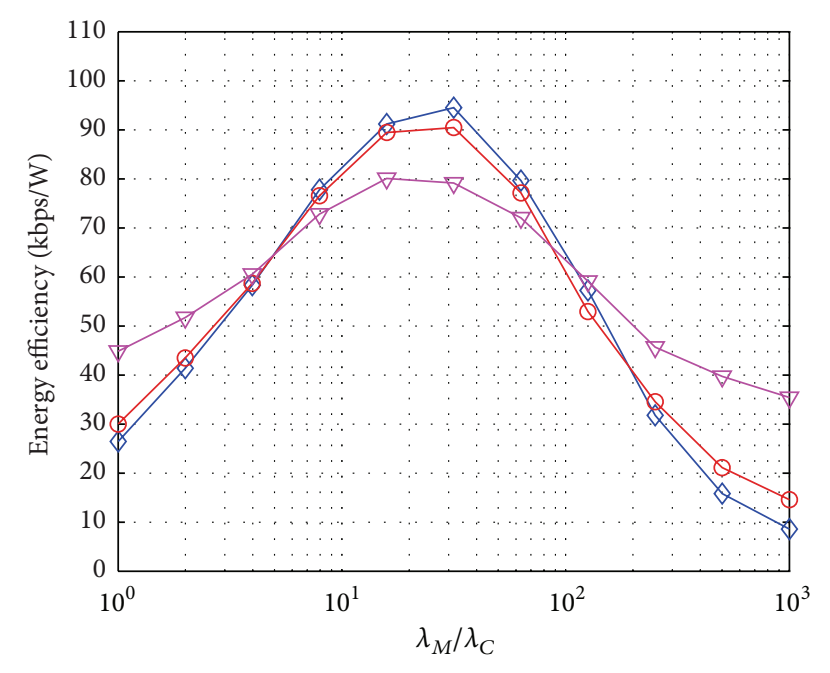

$\neg$ MeNB-load-10\%, by cognition

$\multimap$ MeNB-load- $50 \%$, by cognition

$\rightarrow$ MeNB-load-90\%, by cognition

FIGURE 5: Energy efficiency in cognitive HetNet with different MBS load.

energy efficiency by the deployment of SeNBs. When the density of SeNBs increases to a reasonable level, more spectrum resources utilized by SeNBS bring more performance improvements. However, the energy efficiency is impaired because of the serious intratier interference among SeNBs with amounts of SeNBs located in the network. Then, the cognitive scheme explored with high traffic load of MeNBs is more efficient.

4.3. Energy Efficiency Comparison. With the previous analysis, we can compare the energy efficiency of the proposed cooperative and cognitive schemes in HetNet. For the direct and clear description of the comparison, Figures 4 and 6 are illustrated in Figure 6 together. As shown in Figure 6, it is depicted that the cooperative scheme can outperform the cognitive scheme on the energy efficiency when the density of SeNBs is low. Because some more orthogonal frequency resources can be reused according to the channel quality and interference situation, the utilization of resource is increased and energy efficiency is improved. However, when the number of SeNBs increases to a high value, the cooperative scheme obtains the same performance of energy efficiency as the cognitive scheme. If there is serious interference among the nodes in HetNet, no more spectrum resources can be reused without impairing the energy efficiency in a cooperative manner.

On the other hand, we cannot easily make conclusion that the cooperative schemes are preferred than cognitive schemes for improving the energy efficiency in HetNet. In the proposed schemes, the power consumptions of information exchange in cooperation and spectrum sensing in cognition are not considered. Meanwhile, the complexity of the centralized process in cooperation cannot be neglected. Therefore, the more practical power consumption model

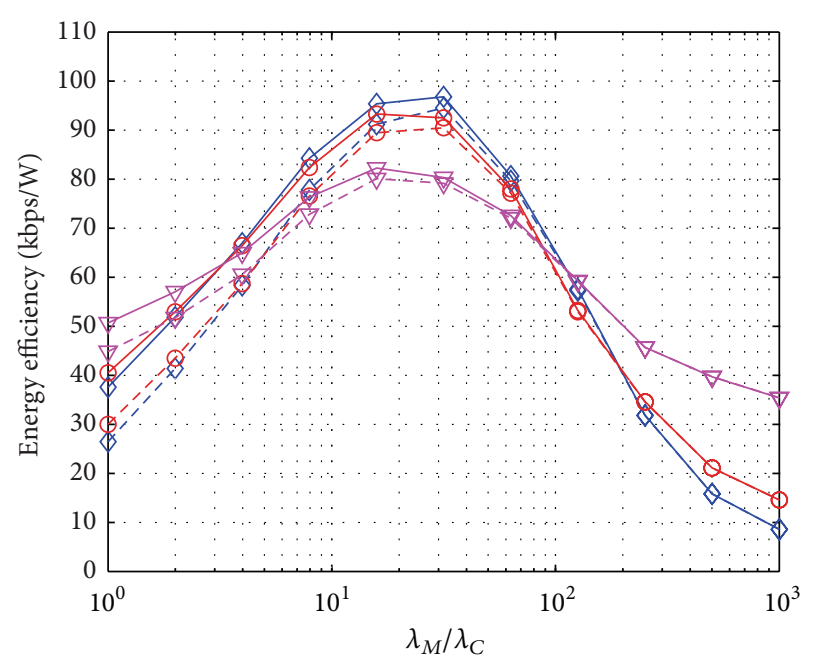

$$
\begin{aligned}
& -\diamond-\text { MeNB-load-10\%, by cognition } \\
& \diamond-\text { MeNB-load-10\%, by cooperation } \\
& -\ominus-\text { MeNB-load- } 50 \% \text {, by cognition } \\
& - \text { - MeNB-load- } 50 \% \text {, by cooperation } \\
& -\nabla-\text { MeNB-load- } 90 \% \text {, by cognition } \\
& -\nabla \text { MeNB-load- } 90 \% \text {, by cooperation }
\end{aligned}
$$

FIGURE 6: Energy efficiency comparison between cooperative and cognitive HetNet with different MBS load.

and cost estimation method in cooperative and cognitive HetNet should be investigated, in order to give some useful suggestions in the design of energy efficient communication networks.

\section{Conclusion}

In this paper, a theoretical framework based on the random spatial models is utilized to analyze the interference characteristic and energy efficiency performance in multitier HetNet. Meanwhile, the performance improvements and interference mitigation with cooperative and cognitive schemes in HetNet are surveyed. In particular, the energy efficiency of the proposed cooperative and cognitive schemes in HetNet is evaluated by the simulation. Furthermore, the comparison between them is presented with different traffic load and network deployment. In future work, more energy efficient cooperative or cognitive schemes will be proposed in HetNet and the practical power and cost model of cooperation and cognition should be investigated to give more reasonable advice in the design of energy efficient wireless communication systems.

\section{Acknowledgments}

This work was supported partially by the State Major Science and Technology Special Projects of China (no. 2013ZX03001001-003), the National Natural Science Foundation of China (no. 61222103), Beijing Natural Science Foundation (no. 4131003), and the New-Star of Science and Technology supported by Beijing Metropolis (xxhz201201). 


\section{References}

[1] M. Peng and W. Wang, "Technologies and standards for TDSCDMA evolutions to IMT-advanced," IEEE Communications Magazine, vol. 47, no. 12, pp. 50-58, 2009.

[2] M. Peng, D. Liang, Y. Wei, J. Li, and H. Chen, "Self-configuration and self-optimization in LTE-advanced heterogeneous networks," IEEE Communications Magazine, vol. 51, no. 5, pp. 36-45, 2013.

[3] B. Han, W. Wang, Y. Li, and M. Peng, "Investigation of interference margin for the co-existence of macrocell and femtocell in OFDMA systems," IEEE System Journal, vol. 7, no. 1, pp. 59-67, 2013.

[4] G. Gür and F. Alagoüz, "Green wireless communications via cognitive dimension: an overview," IEEE Network, vol. 25, no. 2, pp. 50-56, 2011.

[5] N. Himayat, S. Talwar, A. Rao, and R. Soni, "Interference management for $4 \mathrm{G}$ cellular standards," IEEE Communications Magazine, vol. 48, no. 8, pp. 86-92, 2010.

[6] A. Attar and A. H. Aghvami, "A framework for Unified Spectrum Management (USM) in heterogeneous wireless networks," IEEE Communications Magazine, vol. 45, no. 9, pp. 44-51, 2007.

[7] M. Peng, Y. Liu, D. Wei, W. Wang, and H.-H. Chen, "Hierarchical cooperative relay based heterogeneous networks," IEEE Wireless Communications, vol. 18, no. 3, pp. 48-56, 2011.

[8] J. Mitola III and G. Q. Maguire Jr., "Cognitive radio: making software radios more personal," IEEE Personal Communications, vol. 6, no. 4, pp. 13-18, 1999.

[9] Y. Li, L. Zhang, H. Chen, and M. Peng, "Interference coordination in multiple antenna based LTE-advanced heterogeneous systems," International Journal of Antennas and Propagation, vol. 2013, Article ID 167368, 7 pages, 2013.

[10] M. Peng, C. Wang, F. Gao, and W. Xiang, "Advanced antenna technologies in the beyond IMT-advanced systems," International Journal of Antennas and Propagation, vol. 2013, Article ID 156831, 2 pages, 2013.

[11] R. W. Heath Jr., T. Wu, Y. H. Kwon, and A. C. K. Soong, "Multiuser MIMO in distributed antenna systems with out-ofcell interference," IEEE Transactions on Signal Processing, vol. 59, no. 10, pp. 4885-4899, 2011.

[12] M. Z. Win, P. C. Pinto, and L. A. Shepp, "A mathematical theory of network interference and its applications," Proceedings of the IEEE, vol. 97, no. 2, pp. 205-230, 2009.

[13] H. S. Dhillon, R. K. Ganti, F. Baccelli, and J. G. Andrews, "Modeling and analysis of K-tier downlink heterogeneous cellular networks," IEEE Journal on Selected Areas in Communications, vol. 30, no. 3, pp. 550-560, 2012.

[14] H. Jo, Y. Sang, P. Xia, and J. G. Andrews, "Heterogeneous cellular networks with flexible cell association: a comprehensive downlink SINR analysis," IEEE Transactions on Wireless Communications, vol. 11, no. 10, pp. 3484-3495, 2012.

[15] W. C. Cheung, T. Q. S. Quek, and M. Kountouris, “Throughput optimization, spectrum allocation, and access control in twotier femtocell networks," IEEE Journal on Selected Areas in Communications, vol. 30, no. 3, pp. 561-574, 2012.

[16] T. Nguyen, Y. Jeong, T. Q. S. Quek, W. Tay, and H. Shin, "Interference alignment in a poisson field of MIMO femtocells," IEEE Transactions on Wireless Communications, vol. 12, no. 6, pp. 2633-2645, 2013.

[17] R. Xie, F. R. Yu, H. Ji, and Y. Li, "Energy-efficient resource allocation for heterogeneous cognitive radio networks with femtocells," IEEE Transactions on Wireless Communications, vol. 11, no. 11, pp. 3910-3920, 2012.

[18] Y. Pei, Y.-C. Liang, K. C. Teh, and K. H. Li, "Energy-efficient design of sequential channel sensing in cognitive radio networks: optimal sensing strategy, power allocation, and sensing order," IEEE Journal on Selected Areas in Communications, vol. 29, no. 8, pp. 1648-1659, 2011. 

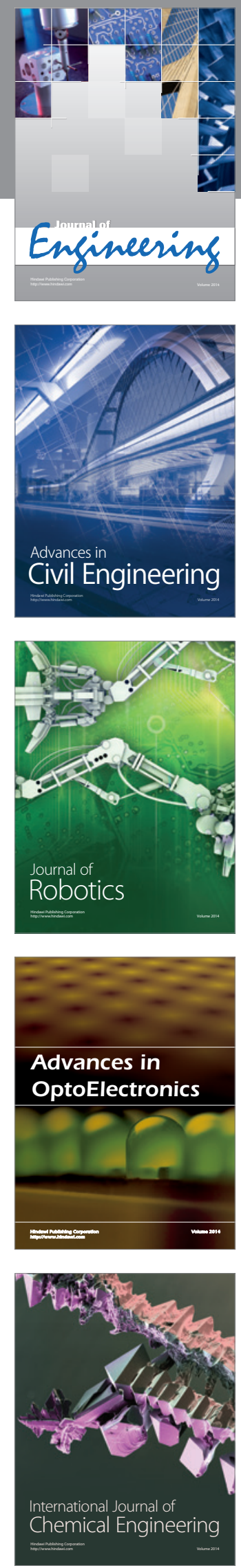

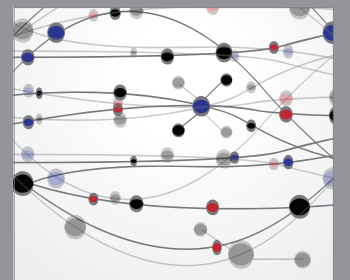

The Scientific World Journal
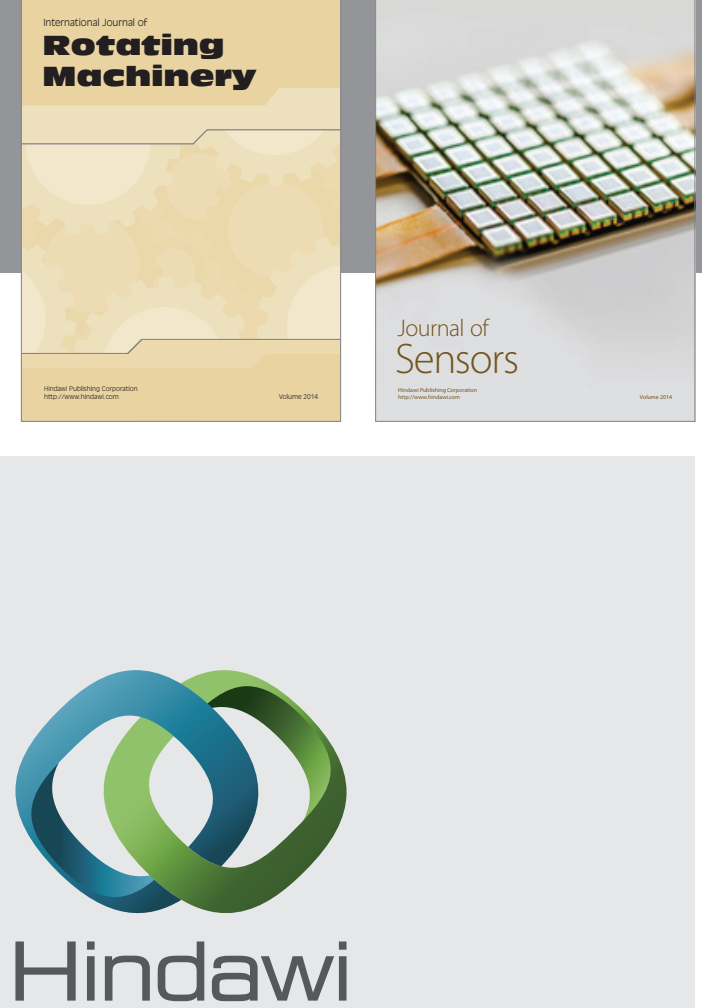

Submit your manuscripts at http://www.hindawi.com
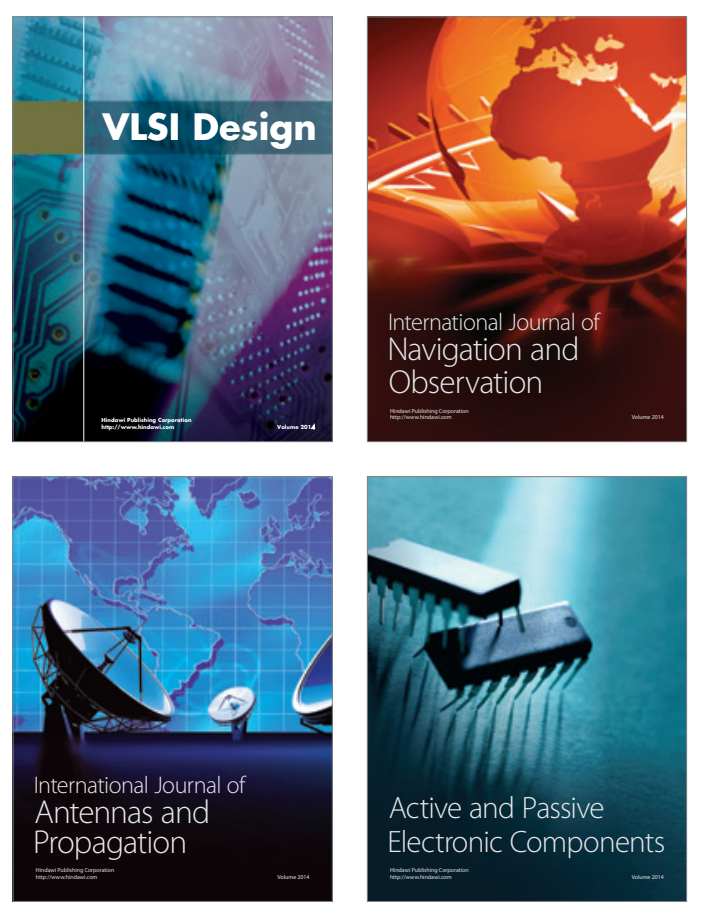
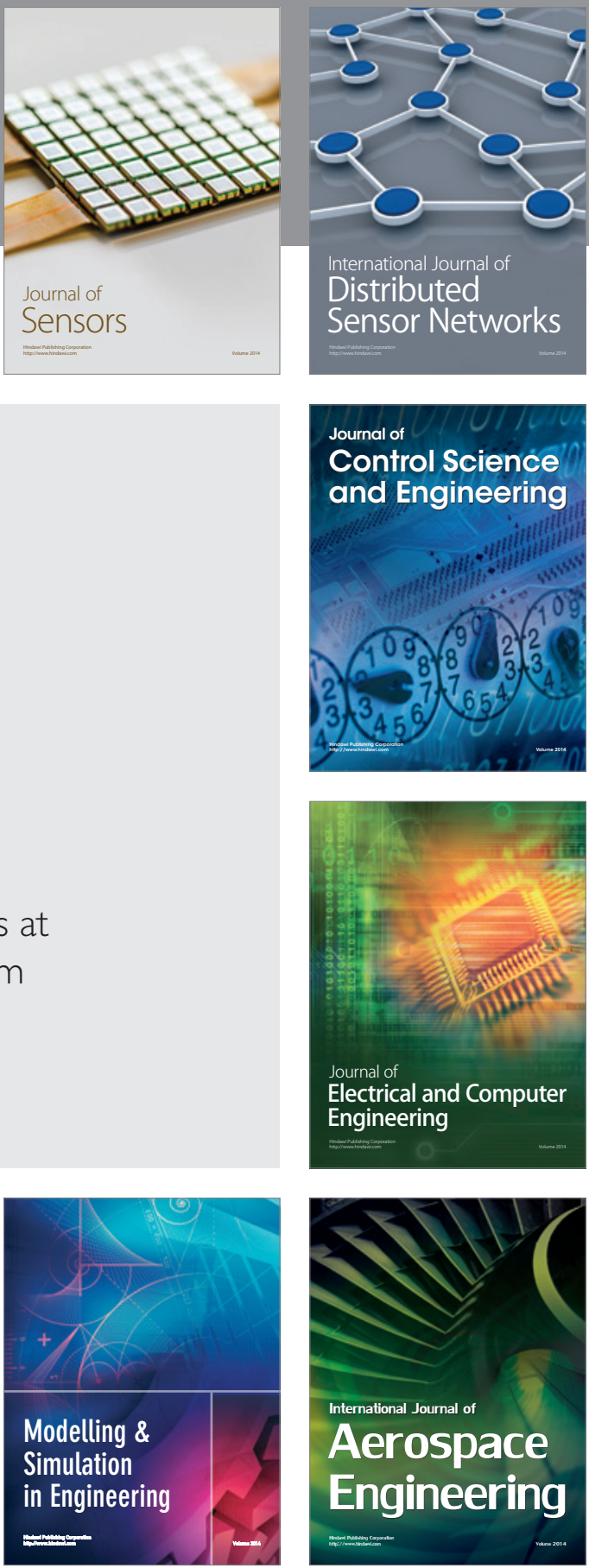

Journal of

Control Science

and Engineering
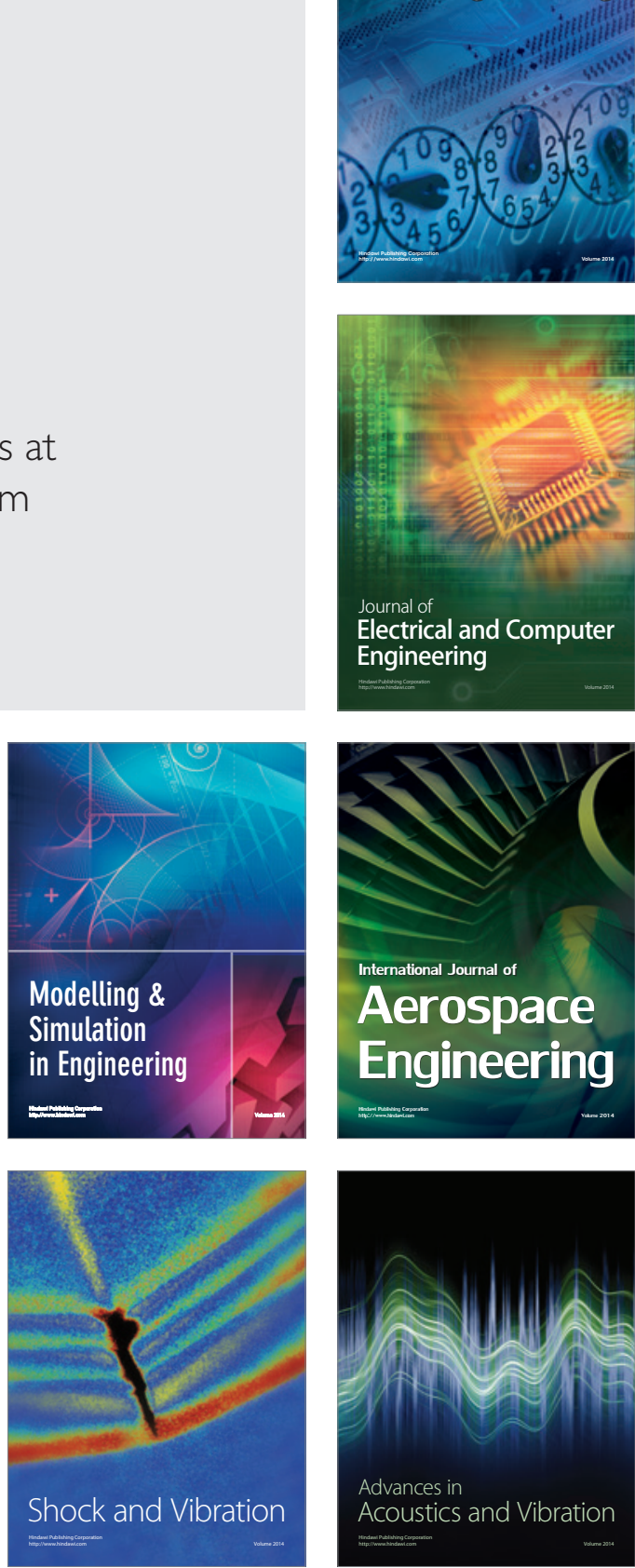